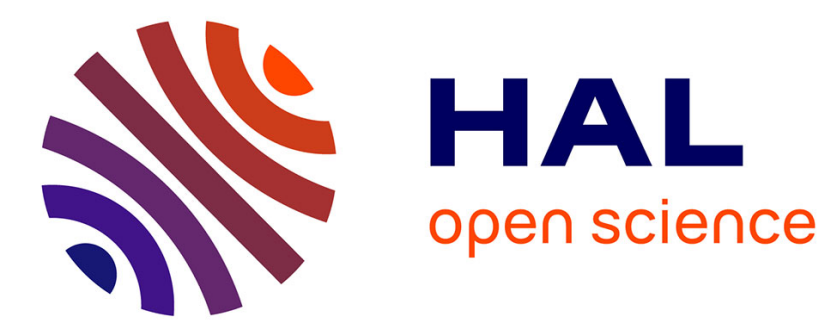

\title{
Le patrimoine pénitentiaire dans le musée d'Histoire de la justice de Criminocorpus (2007-2017)
}

\author{
Marc Renneville, Jean-Lucien Sanchez, Sophie Victorien
}

\section{To cite this version:}

Marc Renneville, Jean-Lucien Sanchez, Sophie Victorien. Le patrimoine pénitentiaire dans le musée d'Histoire de la justice de Criminocorpus (2007-2017). Déviance et Société, 2018, Système pénal et patrimonialisation, 41 (4), pp.619-642. 10.3917/ds.424.0619 . hal-01906911

\section{HAL Id: hal-01906911 \\ https://hal.science/hal-01906911}

Submitted on 26 Mar 2019

HAL is a multi-disciplinary open access archive for the deposit and dissemination of scientific research documents, whether they are published or not. The documents may come from teaching and research institutions in France or abroad, or from public or private research centers.
L'archive ouverte pluridisciplinaire HAL, est destinée au dépôt et à la diffusion de documents scientifiques de niveau recherche, publiés ou non, émanant des établissements d'enseignement et de recherche français ou étrangers, des laboratoires publics ou privés. 


\title{
Le patrimoine pénitentiaire dans le Musée d'histoire de la justice de Criminocorpus
}

(2007-2017)

\author{
Marc Renneville, Jean-Lucien Sanchez, Sophie Victorien
}

\section{Introduction}

Le champ de l'histoire de la justice recouvre un grand nombre de pratiques, de corps d'agents et d'institutions interagissant tout au long d'une chaîne pénale particulièrement dense et étendue (forces de l'ordre, personnels judiciaires et pénitentiaires, tribunaux, établissements pour peine, etc.). En France, certaines de ces institutions ont fait l'objet d'un traitement muséographique qui a pris des formes variées comme le Centre d'exposition Enfants en justice, XIX $X^{e}-X X^{e}$ siècles (École Nationale de Protection Judiciaire de la Jeunesse, Savignysur-Orge), le Musée de la gendarmerie nationale (Melun), le Musée de la préfecture de Police (Paris), la Collection criminalistique de l'École nationale supérieure de la police (Saint-Cyrau-Mont-d'Or), l'espace pédagogique Pierre Cannat de l'École nationale d'administration pénitentiaire (ÉNAP, Agen) ou le Musée du Barreau de Paris. Il n'existe toutefois pas à ce jour de musée national dédié à l'histoire de la justice. En attendant une création souhaitable, le Musée d'histoire de la justice de Criminocorpus remplit cette fonction sur le plan numérique. Ouvert à tout public en accès libre et gratuit, il rassemble thématiquement des reproductions de documents et d'objets conservés dans des lieux différents en assurant une scénographie spécifique. La question du patrimoine pénitentiaire s'y trouve particulièrement bien représentée en raison du partenariat noué avec l'administration pénitentiaire dès l'ouverture du site, en 2005.

L'institution pénitentiaire est elle-même, par son parc immobilier, organiquement liée à la question du patrimoine, en raison de l'origine ancienne de ses établissements les plus emblématiques. De nombreuses maisons d'arrêt situées en centre-ville témoignent d'un moment-clef de l'architecture judiciaire du $\mathrm{XIX}^{\mathrm{e}}$ siècle et les grandes maisons centrales du $\mathrm{XIX}^{\mathrm{e}}$ siècle ont souvent été créées par appropriation d'abbayes (Fontevrault, Clairvaux, Eysses...) ou de châteaux prestigieux (Gaillon, Cadillac...) dont l'architecture est à la fois typique et remarquable. Si la préservation des éléments patrimoniaux de ces lieux n'est pas inscrite dans les missions prioritaires de l'institution, l'idée même de créer un musée des prisons est ancienne. Elle a été portée par Louis Herbette, directeur de l'administration pénitentiaire, lors de l'exposition universelle de 1889 (Herbette, 1891). Il a fallu attendre plus d'un siècle pour qu'un musée pénitentiaire ouvre enfin, en 1995. Malheureusement, cette initiative n'a pas été soutenue dans la durée et le Musée national des prisons de Fontainebleau a définitivement fermé ses portes en 2010. Une partie des collections rassemblée par le Musée est désormais présentée au sein de l'espace pédagogique Pierre Cannat de l'ÉNAP. Cet espace est géré par le centre de ressources sur l'histoire des crimes et des peines (CRHCP), créé au sein de la médiathèque de l'école dans la dynamique de son déménagement à Agen (Renneville, 2004, 2005a). Le Musée national des prisons fut une expérience à la fois remarquable et éphémère. Porté par sa conservatrice Catherine Prade et par l'historien 
Christian Carlier ${ }^{1}$, il se distinguait par un intérêt patrimonial et scénographique de tout premier plan car il se situait dans une ancienne maison d'arrêt construite en 1855 (Prade, 2012). Le parcours d'exposition était organisé au fil des cellules dans lesquelles le public pouvait apprécier une collection portant sur les prisons, les bagnes coloniaux, les colonies pénitentiaires pour enfants, etc. Ce musée dédié aux prisons et à leur histoire permettait ainsi aux visiteurs de découvrir un établissement pénitentiaire et d'en saisir la réalité en arpentant les coursives, les cellules, les cours de promenade «camembert», la chapelle centrale, etc. Utilisé comme espace pédagogique pour les élèves de l'école pénitentiaire alors située à Fleury-Mérogis dans le cadre des cours d'histoire, ce lieu permettait d'articuler de manière didactique un musée à sa collection en assurant la conservation et la valorisation de cette dernière tout en la présentant sur un site qui lui donnait tout son sens. S'il n'existe plus désormais de «prison-musée » en France métropolitaine, cette option muséographique reste bien représentée sur d'autres sites (Hodgkinson, Piché, Walby, Wilson, 2017). Quelques-uns d'entre eux sont d'ailleurs présentés sur Criminocorpus : le site historique du pénitencier d'Eastern State ${ }^{2}$ (Philadelphie) (Elke, 2017a), le Centre d'interprétation de l'architecture et du patrimoine du camp de la transportation de Saint-Laurent-du-Maroni (Guyane) (Bourdeau, Pierre, 2017a) et la plupart des anciens sites des bagnes australiens (Sanchez, 2015).

Les anciens établissements pour peine offrent des espaces spécifiques (cellules, coursives, portes...) qui se prêtent à des scénographies privilégiant la mise en contexte des objets que ces lieux ont bien souvent générés (comme les objets artisanaux produits par des détenus ou «camelote ») ou hébergés (à l'instar de machines à tisser, de « cages à poules », d'uniformes pénaux, de portes de cellules, d'outils de mensurations anthropométriques, etc.). Toutefois, si la transformation d'une prison en un musée pénitentiaire fait assez naturellement sens, la problématique de la scénographie d'un musée numérique se pose en des termes bien différents. Si aucun mur ne vient limiter son extension, elle doit construire l'architecture numérique du site web dans les contraintes d'une médiation par écran. Comment donc exposer et valoriser un patrimoine matériel et immatériel en lien avec l'histoire pénitentiaire via un site web ? Plus particulièrement, en quoi le numérique peut-il permettre d'assurer la sauvegarde d'un patrimoine pénitentiaire aujourd'hui particulièrement menacé ? Quelles sont les possibilités ? Et quelles sont les limites ? Pour répondre à ces différentes questions, nous présenterons dans notre article la genèse de ce musée ainsi que les principales étapes de son élaboration. Puis nous analyserons différents modules et outils qui y sont accessibles en limitant notre propos au seul patrimoine pénitentiaire. Enfin, nous présenterons « HUGO. Patrimoine des lieux de justice », projet collaboratif particulièrement innovant en matière de sauvegarde et de valorisation du patrimoine judiciaire français.

\section{Le projet Criminocorpus}

\footnotetext{
1 Catherine Prade a été conservatrice du Musée national des prisons de 1991 à 2008 (ministère de la Justice, Direction de l'administration pénitentiaire, DAP). Christian Carlier a été directeur d'établissement pénitentiaire et chargé de mission auprès de la DAP. Ses travaux portent sur l'histoire de l'administration pénitentiaire, ses établissements et ses personnels. Il est également le créateur de la revue Histoire pénitentiaire diffusée par la DAP.

2 Eastern State Penitentiary Historic Site.
} 
Criminocorpus est à l'origine la dénomination d'un site web produit dans le cadre d'un projet de recherche sur l'histoire de la criminologie française conduit de 2003 à 2005 (Renneville, 2005b). Nous avons décrit ailleurs l'évolution du site sous l'angle de la numérisation des sources (Renneville, 2017) et au point de vue des humanités numériques (Renneville, Sanchez, Victorien, 2015, 2018). Il n'en sera donc fait ici qu'un bref rappel.

L'objectif de Criminocorpus n'était pas seulement de publier en ligne les résultats de la recherche mais d'initier la construction d'un espace fédérateur constitué de corpus, d'instruments de recherche, d'articles et d'expositions. Il s'agissait ainsi de pallier l'éclatement relatif du domaine de la recherche et des savoirs en matière d'histoire de la justice. Le regroupement sur un site unique de ces différents corpus difficilement accessibles visait à faciliter leur consultation et à favoriser la diffusion de l'histoire de la justice auprès d'un large public. Cet objectif initial demeure toujours celui de Criminocorpus et structure l'identité du site et les différentes innovations qui ont jalonné son histoire.

Lancé le $1^{\text {er }}$ décembre 2005, Criminocorpus se présentait sous la forme d'un portail qui réunissait une revue, des expositions virtuelles et des outils dédiés à la recherche. Un an plus tard il était administré par le Centre Alexandre Koyré (UMR CNRS 8560) et allait connaître à partir de cette date de nombreux changements. En 2008, il s'enrichit d'un carnet (ou blog) dédié à la recherche et changea de logo. Puis son hébergement migra du Centre de calcul de 1'IN2P3 (USR CNRS 6402) à celui de la Très Grande Infrastructure de recherche dédiée aux lettres, aux sciences humaines et sociales et aux humanités numériques (TGIR Huma-Num), mise en œuvre par le ministère de l'Enseignement supérieur et de la Recherche et porté par le CNRS, Aix-Marseille Université et le Campus Condorcet (Paris). De même, la revue scientifique Criminocorpus. Revue hypermédia et le carnet de recherche de Criminocorpus furent respectivement hébergés sur Revues.org (OpenEdition Journals) et Hypothèses dans le cadre d'un partenariat avec le Centre pour l'édition électronique ouverte (CLÉO, UMS CNRS 3287). Mais au bout de sept ans d'activité, l'ajout continuel de nouveaux contenus, les mises à jour technologiques et la séparation du site entre trois entités numériques (un portail, une revue et un carnet de recherche) rendirent la navigation compliquée et l'édition complexe. En 2016, la notion de portail fut donc abandonnée au profit de la mise en place d'un Musée virtuel d'histoire de la justice tout en conservant la revue ${ }^{3}$ et le carnet de recherche ${ }^{4}$. Cette refonte permit de simplifier son usage en le déclinant sous la forme de cinq entrées : «Expositions »; «Visites »; «Collections »; «Repères »; et «Outils». Cette nouvelle organisation du site anticipait également sur l'ouverture à venir d'outils collaboratifs destinés au recueil de données (comme le projet HUGO).

En parallèle, l'équipe chargée d'éditer les contenus du site a été consolidée par la création en septembre 2015 du Centre pour les humanités numériques et l'histoire de la justice (CLAMOR, UMS CNRS 3726). Premier laboratoire d'humanités numériques dédié à l'histoire de la justice, le CLAMOR s'inscrit dans un mouvement déjà ancien d'acculturation de l'informatique aux méthodes de l'historien (Renneville, 2018). Le CLAMOR est composé

\footnotetext{
3 https://journals.openedition.org/criminocorpus/

4 http://criminocorpus.hypotheses.org/
} 
de membres permanents, de membres correspondants et de visiteurs. Comme sa fonction l'indique, il propose ses services à tous chercheurs, institutions ou particuliers qui désirent contribuer à la plateforme Criminocorpus et à l'histoire de la justice. Dans ce cadre, de nombreux partenariats ont été établis sous forme de conventionnement, notamment avec les Archives nationales d'outre-mer (ANOM), la Bibliothèques des littératures policières (BiLiPo), l’ÉNAP, des collectionneurs privés, etc.

Le Musée d'histoire de la justice de Criminocorpus, après une longue genèse, dispose désormais d'une scénographie satisfaisante, au sens où elle permet à ses concepteurs de concilier les exigences de la recherche scientifique avec une restitution et une valorisation publique de ses résultats et de ses sources.

Il est possible de mesurer l'audience portée à l'activité du CLAMOR et de Criminocorpus à travers plusieurs indicateurs. En 2017, la fréquentation du Musée affichait une moyenne de 34580 visites mensuelles ; la revue Criminocorpus est désormais la plus consultée sur sa thématique sur le site Revues.org avec une moyenne de 21500 visites par mois ; et la fréquentation du carnet Criminocorpus atteint en moyenne plus de 45000 visites mensuelles, ce qui le place parmi les cinq blogs les plus visités de la plateforme Hypothèses.org. Criminocorpus est également présent sur les réseaux sociaux et compte en 2018 près de 3700 abonnés sur son compte Facebook et 2300 abonnés sur son compte Twitter. Enfin, le CLAMOR publie depuis sa création une lettre d'information mensuelle diffusée en 2018 à 2500 abonnés ${ }^{5}$. La connaissance des publics du site Musée est l'un des enjeux de son développement. Deux récentes études exploratoires menées en 2017-2018 dans le cadre d'une recherche doctorale en ergonomie cognitive ont permis de dégager une première vue sur la motivation des visiteurs et leurs usages du site. Il en ressort, d'une part, que «la fréquentation principale du musée est portée par des visiteurs désireux de se cultiver » (Papinot, 2018, 46) et, d'autre part, que l'attractivité du site repose sur la variété et la qualité des contenus et une dimension esthétique appréciée qui s'impose comme un héritage du musée physique (Papinot, 2018, 46-48). Dépassant ainsi le cercle initial des chercheurs, des étudiants et des professionnels de l'information, Criminocorpus tend à s'établir comme un lieu de connaissance accessible au-delà de la communauté scientifique dont il émane.

\section{Le patrimoine pénitentiaire : de la conservation à la valorisation}

La valorisation et la sauvegarde du patrimoine carcéral dans le Musée d'histoire de la justice de Criminocorpus reposent sur différents modules, comme les visites virtuelles d'établissements pénitentiaires. Elles s'appuient également sur la mise en accès libre de différentes sources qui permettent la compréhension et la diffusion de l'histoire de ce patrimoine.

\subsection{Un corpus de sources pour l'histoire}

\footnotetext{
5 https://clamor.criminocorpus.org/les/lettres-dinformation/
} 
La mise à disposition de sources relatives à l'histoire pénitentiaire constitue un des actes fondateurs du site Criminocorpus avec la mise en ligne dans la bibliothèque de l'intégralité de la revue des Archives de l'anthropologie criminelle. La publication de ce corpus a été suivie en 2007 par la réalisation d'une chronologie sur l'histoire des prisons et des peines (Carlier, Renneville, 2007 a, b, c, 2009) donnant accès aux textes réglementaires qu'elle listait. À sa suite, d'autres chronologies et plusieurs sources de nature administrative ont été publiées comme les Rapports annuels de l'administration pénitentiaire (1945-1991) ou le Code des prisons (1670-1967), en collaboration avec le CRHCP. Ces mises en ligne permettent ainsi de composer un corpus documentaire unique comprenant des séries parfois difficilement accessibles (et qui peuvent de ce fait constituer un obstacle à la recherche scientifique). Par exemple, en partenariat avec les ANOM, nous avons procédé à la numérisation de l'intégralité de la collection des Notices sur la déportation, la transportation et la relégation à la Guyane et à la Nouvelle-Calédonie, publiées par le ministère des Colonies de 1867 à 1915 (soit 25 volumes). Ces ouvrages délivrent de précieuses informations sur le fonctionnement des bagnes coloniaux puisqu'ils sont constitués d'un rapport annuel accompagné de tableaux statistiques et de textes législatifs. Avant cette opération, il n'existait plus que deux exemplaires complets de cette collection : un conservé au dépôt légal de la Bibliothèque nationale de France $(\mathrm{BnF})$ et un autre conservé aux ANOM. Le premier était totalement hors d'usage du fait de son mauvais état de conservation. Et le second était légèrement incomplet (cinq volumes manquants) et sa consultation imposait de se rendre à Aix-en-Provence. Le CLAMOR et les ANOM ont donc procédé à la numérisation de ces notices grâce à un financement conjoint. En outre, afin de compléter cette collection, les volumes manquants furent commandés par le CLAMOR au Département de la Reproduction de la BnF. L'intégralité de la collection des notices sur les bagnes coloniaux est donc désormais aisément consultable sur Criminocorpus. D'autres opérations de ce type sont prévues dans un proche avenir, comme la numérisation de la revue Le Réveil pénitentiaire. Publiée à partir de 1907, elle constitue la plus vieille revue syndicale pénitentiaire et son édition se poursuit toujours de nos jours à travers le titre Le Réveil Pénitentiaire Justice, édité par le syndicat UFAP UNSa Justice. Cette opération permettra ainsi de pouvoir le consulter à nouveau car l'unique collection (1907-1986) est conservée à la BnF où elle est désormais hors d'usage et donc incommunicable aux lecteurs.

D'autres collections constituées d'objets, de photographies ou d'archives sont également visibles dans la bibliothèque du Musée. C'est par exemple le cas de la collection «Prisons. Objets et documents » présentée par le CRHCP. Lors du déménagement des réserves du Musée national des prisons, ce sont près de 800 objets qui ont été transférés et qui sont pour certains exposés au sein de l'espace pédagogique Pierre Cannat. Cette collection numérique présente une sélection de ces objets et documents. D'autres, comme les collections Léon Collin ou Franck Sénateur, proposent des documents d'archives et des photographies rares ayant trait aux bagnes coloniaux de Guyane et de Nouvelle-Calédonie. Signalons enfin la collection Philippe Zoummeroff ${ }^{6}$ qui permet d'accéder à plus de 6000 images allant du

\footnotetext{
${ }^{6}$ Né le 10 mars 1930, Philippe Zoummeroff est ingénieur de formation. Mécène et collectionneur, il a constitué une collection sur la justice pénale contenant près de 15000 livres et imprimés, 1000 manuscrits et 5000
} 
$\mathrm{XVI}^{\mathrm{e}}$ au $\mathrm{XX}^{\mathrm{e}}$ siècle et à près de 48 heures d'entretiens vidéo relatifs à la justice et à son histoire (Renneville, 2014). Ces fonds, qui sont enrichis au fur et à mesure par leurs propriétaires, permettent au public d'accéder à des collections publiques ou privées exceptionnelles par la diversité et la qualité des matériaux réunis (archives, imprimés, gravures, manuscrits, correspondances, affiches, complaintes, photographies, etc.).

\subsection{Un concept émergent : le « patrimoine carcéral »}

Dès sa création en 2005, Criminocorpus a accordé une part importante de ses contenus à l'histoire et au patrimoine pénitentiaire. Mais la préservation de ce patrimoine n'allait pas de soi et constitue encore de nos jours un sujet relativement neuf. Cette prise de conscience a eu pour moteur, entre autres, l'impulsion donnée par l'historien Jean-Claude Vimont qui a énormément contribué à la connaissance et à la reconnaissance de ce patrimoine. Maître de conférences en histoire à l'Université de Rouen et membre du comité de rédaction de Criminocorpus. Revue hypermédia, Jean-Claude Vimont (1955-2015) était spécialiste d'histoire pénitentiaire. Sa thèse rédigée sous la direction de Michelle Perrot portait sur l'histoire de la détention politique en France du XVIII ${ }^{\mathrm{e}}$ au XX ${ }^{\mathrm{e}}$ siècle (Vimont, 1993) et il est l'auteur d'un ouvrage de référence sur l'histoire des prisons françaises (Vimont, 2004). Dans le cadre du master patrimoine qu'il dirigeait à l'Université de Rouen, il a encouragé ses étudiants à s'intéresser au patrimoine pénitentiaire et les résultats de ces travaux ont ensuite été publiés à partir d'octobre 2006 dans une rubrique «Patrimoine carcéral des régions françaises » qu'il dirigeait et animait dans le carnet de recherche de Criminocorpus (Vimont, 2012). L'en-tête de cette rubrique, rédigée par ses soins, indique :

«L'univers carcéral français connaît de profondes mutations en ces premières années $\mathrm{du} \mathrm{XXI}^{\mathrm{e}}$ siècle. De vieux établissements sont promis à la destruction. Cette rubrique offre des informations sur leur passé, sur leur devenir, sur leur reconversion ou leur destruction. Elle propose une réflexion sur le concept nouveau de "patrimoine carcéral". »

Pour définir ce «concept nouveau de "patrimoine carcéral" », Jean-Claude Vimont partait du constat des nombreuses fermetures d'établissements pénitentiaires programmées au cours des années 2000 (Vimont, 2008) suite, notamment, à l'introduction des nouvelles règles pénitentiaires européennes adoptées par la France en 2006. L'administration pénitentiaire a décidé de faire du respect de ces règles «un objectif prioritaire en ce qui concerne l'orientation de sa politique de modernisation $^{7} »$, et elles ont donc eu une incidence conséquente sur les établissements pénitentiaires, qu'ils soient de construction ancienne ou nouvelle. Par exemple, une cellule du «quartier bas » de la maison d'arrêt de Paris la Santé, établissement bâti en 1867 , mesurait $7 \mathrm{~m}^{2}$ en 2015 , date de sa fermeture pour une longue campagne de travaux prévue pour s'achever en 2018. Ces travaux de modernisation ont entraîné l'agrandissement des cellules pour qu'elles atteignent une taille moyenne de $9 \mathrm{~m}^{2}$ (Agence pour l'immobilier de la justice, s.d.). Bien que ces nouveaux standards soient indispensables pour garantir un hébergement décent aux détenus, il n'en demeure pas moins

images rares (gravures, dessins, photographies, etc.). Cette collection a fait l'objet en 2014 et en 2016 d'une vente aux enchères à l'hôtel Drouot (Bergé, 2014 et 2016).

7 http://www.justice.gouv.fr/europe-et-international-10045/les-regles-penitentiaires-europeennes-10283/ 
qu'ils affectent irrémédiablement un grand nombre d'établissements qui se voient dénaturés, lorsqu'ils ne sont pas tout simplement détruits.

Souvent construites au $\mathrm{XIX}^{\mathrm{e}}$ siècle, ces prisons sont devenues vétustes et les conditions d'incarcération qu'elles offrent aux détenus ont fait l'objet de multiples condamnations de la France par la Cour européenne des droits de l'homme. De manière similaire au processus de désindustrialisation qui a permis l'émergence du concept de patrimoine industriel (Daumas, 1980), le mouvement de fermeture, de restructuration et de destruction d'établissements pénitentiaires qui a accompagné la modernisation récente du parc carcéral français a favorisé l'émergence du « concept nouveau de "patrimoine carcéral" » que Jean-Claude Vimont a œuvré à faire connaître et reconnaître (Vimont, 1997).

\subsection{Un dispositif original : les visites de prisons}

Contribuer à la sauvegarde de ce patrimoine confronte l'historien, tel l'ethnologue, à une situation d'urgence et une réflexion sur les mesures à prendre: la conservation des édifices à l'identique n'étant pas envisageable, que faut-il préserver de ces lieux d'enfermement rarement valorisés dans l'imaginaire collectif? À l'occasion des Journées européennes du patrimoine 2014, un collectif d'historiens et d'architectes lança un appel dans les colonnes du quotidien Libération pour alerter sur la situation des anciennes prisons et du péril menaçant le «patrimoine sombre » français (Faure, 2014). La demande était moins de défendre la conservation des lieux que de trouver les moyens d'en préserver la mémoire par un recueil de sources adapté. En effet, des établissements pénitentiaires ferment chaque année et sont remis par le ministère de la Justice à la Direction de l'immobilier de l'État, qui est chargée de leur cession. Ces vieux établissements, souvent hérités du XIX ${ }^{\mathrm{e}}$ siècle (et au-delà), se situent pour beaucoup en centre-ville et attisent ainsi l'intérêt des promoteurs immobiliers. Bon nombre d'entre eux, comme la maison d'arrêt du Havre (Arlaud, Carpentier, Louveau, Mercier, Renneville, Vimont, 2015) ont été détruits ou ont été considérablement remaniés, comme la maison d'arrêt de Bourg-en-Bresse reconvertie en logements (Bertrand, 2016). La précarité et la fragilité de ces vestiges nous impose ainsi d'agir la plupart du temps dans l'urgence et avec des moyens limités.

\section{Une réalisation pionnière : la visite de la maison d'arrêt du Havre}

Le souhait d'expérimenter de nouvelles écritures numériques associé à la volonté de contribuer à la sauvegarde des pratiques professionnelles pénitentiaires nous ont conduit à concevoir le projet d'une visite de prison en ligne dans le cadre du programme de recherche collectif ANR SCIENCEPEINE (Sciences, savoirs et politique de l'exécution des peines en France, 2009-2014) (Renneville, 2014). Le choix de l'établissement du Havre a été conditionné par une fermeture imminente. Située au 25 rue Lesueur dans le centre-ville, à proximité de la gare, l'ancienne maison d'arrêt du Havre a fermé ses portes en 2010 suite à l'ouverture d'un nouveau centre pénitentiaire de 690 places construit sur les communes de Gainneville et de Saint-Aubin. Installé désormais à 15 kilomètres à l'est du Havre, ce nouveau centre pénitentiaire était destiné à accueillir les détenus de la maison d'arrêt du Havre qui a été intégralement démolie en 2012. 
Construite en 1860, la maison d'arrêt disposait d'une particularité architecturale : celle de contenir des dortoirs et des cellules. La détention s'organisait initialement autour de trois dortoirs destinés à accueillir 180 détenus. Puis il y fut adjoint une aile cellulaire au début du $\mathrm{XX}^{\mathrm{e}}$ siècle. Cet aménagement s'inscrivait dans un contexte d'individualisation des peines qui a connu de nombreux changements législatifs au cours du XIX ${ }^{\mathrm{e}}$ siècle. Ainsi, à travers l'évolution architecturale de cette prison peuvent se lire et s'appréhender près de deux siècles d'histoire pénitentiaire et leurs principales évolutions.

Notre projet de visite en ligne visait toutefois à dépasser la restitution de l'histoire du lieu par son architecture. L'idée de ce module de visite virtuelle est effectivement née d'échanges en 2010 entre un ethnologue, Jean Arlaud, et un historien, Marc Renneville, qui avaient été tous deux membres du Laboratoire Anthropologie visuelle et sonore des mondes contemporains (EA 3504), à l'Université Paris 7. De ces échanges est née l'intention de croiser les méthodes de recherche pour donner à voir un établissement pour peine de l'intérieur, à travers le regard et la parole de ceux qui en sont les acteurs et qui l'animent au quotidien. L'objectif était d'initier un recueil de données sur place afin de produire une restitution numérique originale, donnant simultanément accès à des ressources historiques et à des témoignages filmés explicitant des pratiques professionnelles dans un lieu habituellement soustrait au regard du public. La démarche de travail reposait sur le recueil filmique de témoignages des personnels de l'établissement invités à présenter leur lieu de travail et son fonctionnement. Le fait de privilégier ce type de témoignages présentait l'intérêt que le visiteur soit accueilli et accompagné tout au long de son parcours par des surveillants, c'est-àdire par ceux qui connaissent le mieux leur établissement. Ces derniers, en outre, l'évoqueraient avec leurs propres mots au fil d'une visite où seraient exposés les principaux bâtiments de la détention avant leur disparition. En effet, les pratiques des personnels pénitentiaires, à l'instar de leurs établissements, évoluent et disparaissent avec la destruction de ces vieux bâtiments et l'ouverture de nouveaux, d'une architecture et d'un fonctionnement très différents. La conception du module entendait répondre à trois usages possibles. La visite devait permettre aux agents ayant travaillé dans une prison donnée de pouvoir en conserver la trace et la mémoire. Elle pouvait également être mobilisée dans le cadre de formations dispensées aux élèves de l'ÉNAP. Enfin, elle était une réalisation à visée civique, permettant aux citoyens de découvrir une prison et d'appréhender la réalité carcérale à partir du point de vue d'un personnel pénitentiaire.

Mais si cette idée était stimulante, il n'en restait pas moins à la matérialiser sous la forme d'une réalisation numérique. Le matériau de ce module reposait essentiellement sur des séquences filmées du personnel de l'établissement. Pour des raisons d'ordre juridique, il n'a pas été possible de réaliser le même type de séquences avec des détenus. En accord avec la Direction interrégionale des services pénitentiaires (DISP) du Grand-Ouest et la direction de l'établissement, les prises de vue furent réalisées à la maison d'arrêt du Havre entre les mois de mars et d'avril 2010. Elles furent ensuite montées et implémentées dans un module qui se présente sous la forme d'une vue aérienne de la maison d'arrêt. Le visiteur dispose sur cette interface d'un menu depuis lequel il peut sélectionner un des différents niveaux du bâtiment (caves, rez-de-chaussée, premier étage, deuxième étage, etc.). Puis les bâtiments ouverts à la 
visite apparaissent en surbrillance. Il suffit alors de cliquer sur l'un d'entre eux pour atteindre une fenêtre depuis laquelle on peut accéder à une vidéo ainsi qu'à des photographies du bâtiment présenté. Pour prolonger sa visite, le visiteur peut également accéder à des galeries présentant des plans de l'établissement provenant du CRHCP, à des vues aériennes (de 1939 à 2014) et à une rubrique qui rassemble plusieurs contenus du site (Musée, revue et carnet de recherche) en lien avec l'histoire des prisons (collections, expositions, articles, chronologies, etc.).

\section{La visite de la maison d'arrêt de Paris la Santé}

La réalisation suivante concerna la maison d'arrêt de Paris la Santé (Renneville, Sanchez, Victorien, Vimont, 2015). La possibilité de tournage fut ici encore la conséquence d'une opportunité de fermeture. Cette maison d'arrêt, la dernière située encore dans Paris intra-muros, a fermé ses portes en juillet 2014 pour une importante campagne de travaux de modernisation. À cette occasion, la direction de la maison d'arrêt de Paris la Santé, le service pénitentiaire d'insertion et de probation de Paris, le Cercle culturel de la maison d'arrêt de Paris la Santé, la DAP et la DISP de Paris décidèrent l'ouverture de ses portes au public lors des Journées européennes du patrimoine 2014. Cet événement inédit rencontra un très grand succès public avec plus de 1000 visiteurs inscrits sur deux jours.

L'équipe de Criminocorpus a réalisé une visite portant sur une grande partie de l'établissement. L'étape du prototype étant dépassée, l'interface de visualisation offre depuis la visite de la prison de la Santé une structure relativement standardisée, intuitive et simple d'usage, à l'image des autres modules d'assemblage du Musée (comme ceux des expositions virtuelles, des collections ou des chronologies). Il nous semble toutefois important d'insister sur la nécessaire complémentarité des ressources numériques en ligne sur le site. Ainsi, pour la prison de la Santé, le site propose à la fois une visite et une exposition virtuelle consacrée à l'histoire de l'établissement (Soppelsa, 2015). La page d'accueil des visites permet aux visiteurs de poursuivre leur navigation vers d'autres modules présentés sur Criminocorpus et déclinés sous deux formes : «Histoire» et «Témoignages ». Le premier renvoie à de nombreux contenus (expositions, documents, chronologies, articles, etc.) en lien avec l'histoire des prisons. Le second renvoie à des témoignages de personnels pénitentiaires (directeurs de l'administration pénitentiaire, directeurs d'établissement, détenus, journaux de détention, photographies, etc.). Ainsi ce module de visite virtuelle constitue pour le public une porte d'entrée qui lui permet de découvrir d'autres contenus édités sur Criminocorpus (Musée, revue et carnet de recherche) vers lesquels il ne se serait pas forcément orienté au début de sa navigation.

Si les visites multimédia proposées par Criminocorpus sont susceptibles d'éveiller l'intérêt du public et de le sensibiliser au patrimoine pénitentiaire, elles ne sauraient être l'unique réponse possible face aux menaces et aux destructions dont il est l'objet aujourd'hui. En outre, ces tournages ne sont pas aisés à réaliser car il est parfois trop tard pour accéder à certains établissements fermés ou en cours de fermeture et beaucoup se situent dans des régions éloignées. La question de la temporalité joue contre la possibilité de multiplier l'expérience car les conditions de filmage sont très difficiles à réunir. En effet, il faut concilier 
la certitude d'une fermeture avec une possibilité de filmer un établissement en situation «normale », ce qui n'est, de fait, plus le cas lorsque l'événement qui autorise le film est connu des personnels de l'établissement. La réalisation d'une visite de prison vide présente un faible intérêt car sa dimension de patrimoine vivant est perdue. Plus important encore, la vue des lieux ne peut que biaiser l'interprétation que pourraient en avoir les visiteurs. Dans un établissement désaffecté, le sol des cellules et des couloirs est souvent jonché d'objets ou de documents abandonnés, la poussière s'est déposée partout et, surtout, la prison est silencieuse. Or, le paysage sonore d'une détention fait partie intégrante de son identité, voire même de son fonctionnement. Les murs de la détention constituent une caisse de résonance qui amplifie les bruits continuels de clefs, de pas, de mouvements de grilles, de sirènes, de paroles et de cris des détenus et des personnels de surveillance qui alimentent une ambiance sonore très spécifique. Nous avons réalisé deux visites virtuelles d'établissements pénitentiaires désaffectés : celles des maisons d'arrêt de Chartres et Saint-Michel de Toulouse. Bien qu'elles n'aient pas encore été montées et mises en ligne, les différentes prises de vue réalisées sont assez décevantes. Lorsque nous nous sommes rendus en 2014 à la maison d'arrêt SaintMichel de Toulouse (qui a fermé ses portes en 2009), celle-ci était totalement à l'abandon. Mais cet établissement était devenu dans l'intervalle un terrain d'entraînement pour les équipes régionales d'intervention et de sécurité de l'administration pénitentiaire et l'intérieur était, outre sa vétusté, très altéré par ce nouvel usage. Quant à la maison d'arrêt de Chartres, nous avons réalisé les séquences peu de temps après sa fermeture, en 2014. Mais le désordre causé par sa récente évacuation biaise trop fortement notre intention de restitution du lieu.

\section{La visite de la maison d'arrêt de Caen}

L'idéal est donc de procéder à un recueil de données en amont. Ce fut le cas par exemple de la réalisation en 2016 de la visite virtuelle de la maison d'arrêt de Caen. Cette initiative s'inscrivait dans le cadre d'un programme de recherche piloté initialement par JeanClaude Vimont. Lancé à son initiative, le projet scientifique «Patrimoine carcéral normand » (PCN) est financé par la Région Normandie (2016-2018) dans le cadre de la politique des Grands Réseaux de Recherche et coordonné à l'Université de Rouen. Ce programme prévoyait la réalisation de deux visites multimédia : celles des maisons d'arrêt de Caen et d'Alençon. En ce qui concerne cette dernière, ancien Château des Ducs d'Alençon, elle a fermé ses portes en 2009. La maison d'arrêt de Caen est toujours en activité et son directeur a répondu favorablement à notre proposition. Construite entre 1899 et 1904, sa fermeture a été officiellement évoquée en 2013 par la ministre de la Justice, Michèle Alliot-Marie, pour 2017, puis a été repoussée sine die. Néanmoins, voulant profiter du laps de temps dont nous disposions grâce au financement offert par le projet PCN, nous avons décidé d'entreprendre la réalisation d'une visite virtuelle préventive. Nous avons réalisé des séquences à la maison

d'arrêt de Caen en vue de leur mise en ligne à moyen ou long terme. Pour des raisons de sécurité, nous ne pouvons effectivement pas diffuser auprès du public cette visite virtuelle tant que la prison demeurera en activité et nous ne pourrons donc le faire que lorsqu'elle aura été totalement fermée.

Pour réaliser les séquences, nous nous sommes adjoint les services d'un réalisateur spécialisé dans le tournage et le montage de documentaires scientifiques, accompagné d'une 
preneuse de son. Après une journée passée en repérage, nous avons pu disposer de deux jours consécutifs sur place pour réaliser le tournage. Accompagné de deux surveillants ayant réalisé pratiquement toute leur carrière dans cet établissement, nous avons filmé la quasi-intégralité de la détention. Puis nous avons procédé de même dans le bâtiment réservé aux femmes détenues où une surveillante a également accepté de témoigner. Ce modèle de réalisation préventive de visites virtuelles semble empiriquement le plus adéquat et celui qui se prête le mieux à une certaine reproductibilité de nos visites. Mais il a l'inconvénient d'être étroitement dépendant des financements que nous serons susceptibles d'obtenir pour pouvoir l'employer à une échelle et une fréquence plus importantes. D'autre part, ce modèle est adapté pour les établissements anciens, certes, mais vétustes et dont la fermeture semble inéluctable. C'est loin d'être le cas pour tous aujourd'hui. Ainsi, beaucoup de vielles prisons, qui représentent pourtant un intérêt patrimonial de premier ordre, comme le centre pénitentiaire de Fresnes (ouvert en 1898) ou la maison centrale d'Ensisheim (ouverte en 1811), sont toujours en activité et le seront encore durant de nombreuses décennies. De ce fait, nous ne pouvons entreprendre des opérations préventives de visites virtuelles de ces établissements si nous demeurons dans l'incapacité de savoir si elles pourront un jour être mises en ligne sur Criminocorpus.

\section{Un écosystème numérique au service du patrimoine}

La valorisation et la sauvegarde du patrimoine carcéral dans le Musée d'histoire de la justice de Criminocorpus ne reposent pas sur les seules visites multimédia, mais également sur les expositions virtuelles et, depuis peu, sur le projet collaboratif « HUGO. Patrimoine des lieux de justice ».

\section{Les expositions virtuelles}

Toujours dans l'optique de réunir en un lieu unique des savoirs en lien avec l'histoire de la justice disséminés dans des lieux différents, le CLAMOR opère une veille sur les expositions en lien avec l'histoire judiciaire, invite des contributeurs réguliers ou nouveaux mais peut aussi être sollicité directement par une institution ou un particulier. À ce titre, le Musée compte actuellement 39 expositions virtuelles et leur nombre continue de croître. Elles se répartissent au sein de plusieurs thématiques : «Prisons », «Bagnes », «Art et justice», «Suspects, accusés, coupables » et «Peine de mort». Les thématiques «Prisons» et «Bagnes » regroupent 21 expositions issues de contributeurs très divers. Certaines sont des expositions présentées dans des musées et adaptées a posteriori pour être mises en ligne dans le Musée. C'est le cas par exemple de l'exposition « À l'ombre des murailles. Quand le château était prison », présentée au Musée du château de Mayenne du 22 novembre 2014 au 20 septembre 2015. Le château de Mayenne fut effectivement une prison de la fin du XVII siècle jusqu'en 1934. Cédé à la municipalité de Mayenne deux ans plus tard, il devint en 2008 un musée dédié à des collections municipales et départementales. Grâce à cette exposition, l'équipe du musée a mis en valeur le passé carcéral du château et a présenté au public de nombreux objets conservés dans ses réserves en lien avec cette période d'activité. Le CLAMOR a proposé une déclinaison en ligne de cette exposition qui repose essentiellement sur la présentation de documents transmis par les Archives départementales de la Mayenne 
(Musée du château de Mayenne, 2017). Ainsi, le module d'exposition virtuelle permet d'affranchir des expositions «physiques» de leurs limites tout à la fois spatiales et temporelles.

D'autres expositions peuvent également nous être proposées par des chercheurs ou des personnes simplement intéressées par l'histoire de la justice. L'exposition «La prison de Bicêtre », réalisée par les historiens Jean-Claude Farcy et Laurence Guignard, constitue un exemple très intéressant de valorisation d'une recherche scientifique (Farcy, Guignard, 2015a). Richement illustrée et adossée à un dépouillement exhaustif d'archives, elle prolonge un premier travail réalisé par les auteurs et édité sous la forme d'un article dans une revue scientifique (Farcy, Guignard, 2015b). En complément, elle s'accompagne d'une base de données recensant tous les détenus administratifs incarcérés dans cette prison entre 1813 et 1835. Le module d'exposition virtuelle permet également de valoriser des travaux scientifiques en lien avec le patrimoine pénitentiaire en les diffusant auprès du public, mais sous une forme didactique.

Enfin, certaines expositions peuvent également provenir d'articles publiés dans des revues ou des sites web. Par exemple, le volume 11 de la revue Histoire pénitentiaire, publiée par la DAP et hébergée sur le carnet de recherche de Criminocorpus, était consacré au patrimoine et à l'architecture pénitentiaire (Gaume, Sanchez, Victorien, 2016). Parmi les articles reçus, certains étaient très richement illustrés comme ceux de Sara Jane Elke sur le pénitencier d'Eastern State (Elke, 2017b) ou de Marie Bourdeau et de Michel Pierre sur le camp de la transportation de Saint-Laurent-du-Maroni (Bourdeau, Pierre, 2017b). Mais l'édition en ligne dans le carnet de recherche ne permet pas d'utiliser beaucoup de photographies. Afin d'y remédier, trois de ces articles ont été modifiés afin d'être présentés sous la forme d'une exposition virtuelle, comme celle de «La maison centrale de Fontevraud » (Stalder, Mathurin, 2017).

Ces expositions sont la plupart du temps «fermées», bien que leurs auteurs conservent la possibilité de les enrichir ou de les modifier ultérieurement. Cependant d'autres demeurent « ouvertes », comme l'exposition «La mémoire des murs », coordonnée par JeanClaude Vimont (Breton, Bulmé, Farcy, Pitte, Poulain, Pouvreau, Renneville, Sanchez, Tronel, Veyret, Vimont, Zaidman, 2014). Cette exposition représente une contribution remarquable à la connaissance et à la conservation du patrimoine carcéral, en portant sa focale sur les graffitis de détenus. Conscient de leur fragilité, Jean-Claude Vimont a beaucoup œuvré à la sauvegarde de ces «archives sensibles » et réalisé des campagnes de recueil dont le résultat est visible dans cette exposition (comme les graffitis de la colonie pénitentiaire des Douaires ou ceux de la maison d'arrêt de Paris la Santé). Cette exposition compte actuellement treize chapitres et continue d'en accueillir de nouveaux au fur et à mesure.

À l'image des visites virtuelles, le module d'exposition virtuelle a également été l'objet d'une évolution technologique qui a permis d'améliorer considérablement son usage et ses performances puisqu'il nous permet d'exploiter désormais un nombre illimité d'images.

Les expositions virtuelles du Musée d'histoire de la justice constituent le contenu le plus consulté sur Criminocorpus. Néanmoins, comme les visites virtuelles, ce module 
présente également quelques limites. D'une part, malgré la veille assurée par le CLAMOR (et les signalements qui nous sont transmis), certaines institutions ne donnent pas toujours suite à nos propositions de collaboration. D'autre part, la plupart des expositions «physiques »ne peuvent pas être déclinées telles quelles en version numérique. De nombreux objets qui figurent dans les parcours d'exposition initiaux, de par leur taille par exemple, ne peuvent pas être intégrés. En outre, ces expositions «physiques » ne sont pas élaborées en amont en envisageant leur possible déclinaison numérique. La scénographie originale ne peut donc pas être restituée à l'identique, même si l'exposition numérique s'efforce, en règle générale, de conserver le découpage d'origine. Un autre problème se pose également avec la question des droits d'auteurs. Certains collectionneurs privés ou institutions, s'ils ont accepté de prêter un document ou un objet original dans le cadre d'une exposition "physique », n'acceptent pas forcément de céder leurs droits sur leur copie numérique. À l'inverse, il arrive fréquemment que nous enrichissions le contenu de ces expositions grâce à l'ajout d'images ou de documents d'archives issus de fonds d'archives publiques, comme celui du portail Gallica ou grâce aux partenariats dont nous disposons avec les Archives nationales ou certains centres d'archives départementales. Les droits de réutilisation non commerciale des archives publiques (considérées comme des «informations publiques ») nous autorisent effectivement à les exploiter sur Criminocorpus sous réserve de respecter le droit de la propriété intellectuelle (et d'indiquer lisiblement la source du document). Mais les quelques limites exposées ci-dessus pèsent toutefois peu au regard de la valeur ajoutée apportée au Musée d'histoire de la justice par ces multiples contributions. Car comme tout musée, celui-ci se doit de présenter des expositions et de les renouveler fréquemment pour maintenir l'intérêt de ses visiteurs. Néanmoins, le fait qu'il soit numérique permet d'en présenter conjointement 39 et ce sans limite de durée.

Le recensement du patrimoine pénitentiaire: un travail de recueil collectif via le projet «HUGO. Patrimoine des lieux de justice »

Le musée s'est enrichi en 2017 d'un outil collaboratif destiné à dresser un inventaire du patrimoine judiciaire français intitulé «HUGO. Patrimoine des lieux de justice ${ }^{8}$ Cette initiative est en partie inspirée par la base de données «Les monuments aux morts. FranceBelgique » réalisée et mise en ligne par l'Institut de Recherches Historiques du Septentrion (UMR CNRS 8529). ${ }^{9}$ Ce site a pour vocation de recenser tous les monuments aux morts français et belges et il est renseigné par des auteurs qui soumettent des fiches modérées par l'équipe. HUGO poursuit un objectif similaire dans le domaine du patrimoine judiciaire français (tribunaux et établissements pour peine). Ce service est une œuvre scientifique collective (en conformité avec l'article L113-2 du Code de la propriété intellectuelle) coordonnée par le CLAMOR qui la publie, la développe, en assure la pérennité et la rend librement accessible en ligne à des fins de connaissances et de partage. Soutenu par la Mission de recherche Droit et Justice, HUGO est réalisé en partenariat avec les Archives nationales, la région Normandie, le Groupe de Recherche d'Histoire de l'Université de Rouen

\footnotetext{
8 https://hugo.criminocorpus.org/fr/

${ }^{9}$ http://monumentsmorts.univ-lille3.fr/
} 
(GRHis, EA 3831), le Centre d'Histoire Judiciaire de 1'Université de Lille 2 (UMR CNRS 8025), l'équipe d'accueil Interactions, Transferts et Ruptures artistiques et culturels (InTRu, EA 6301) et l'ÉNAP.

De nombreux établissements pour peine et tribunaux en activité, désaffectés, requalifiés ou disparus quadrillent les départements qui sont les relais à l'échelon local de l'administration judiciaire. Malgré leur ancrage dans les territoires, les lieux de justice demeurent un patrimoine encore largement méconnu. HUGO constitue donc un service ouvert à tous et dédié à la connaissance partagée de ce patrimoine. Il vise à dresser un inventaire des lieux de justice en rassemblant des informations relatives à leur architecture, leur histoire et leur mémoire. Conçu sur une base participative, il offre à chacun la possibilité de contribuer à la collecte des données. Moyennant l'acceptation de ses termes et conditions d'utilisation, il s'adresse aux chercheurs, étudiants, conservateurs, membres de sociétés savantes, etc. et à tout particulier intéressés par cette thématique. Il s'adresse également à des institutions, comme des centres d'archives ou des bibliothèques, qui peuvent y participer en établissant un partenariat avec le CLAMOR. Les contributeurs peuvent soit compléter un lieu existant déjà dans la base, soit proposer la création d'un nouveau lieu. Les membres de l'équipe accompagnent les contributeurs pour les assister durant le processus d'élaboration de leur fiche qui est ensuite implémentée dans une base de données se présentant sous la forme d'une carte géographique. Le visiteur peut soit interroger cette base soit cliquer sur un lieu situé sur la carte pour accéder à sa fiche. Celle-ci propose alors les champs suivants : carte du lieu (avec son adresse); «Identité »; «Dates-clés »; «Personnes liées à l'établissement»; «Statistiques »; et « Ressources ». Tout contributeur peut ensuite s'il le souhaite compléter ultérieurement cette fiche en y apportant des informations supplémentaires.

La constitution de cette base de données vise à établir, à terme, un état précis du patrimoine judiciaire français. Afin d'amorcer son renseignement, 103 notices d'établissement ont déjà été rédigées par les membres de l'équipe et d'autres le seront au fur et à mesure. Un partenariat a également été établi avec les Archives départementales de l'Ain dont l'équipe va renseigner les lieux de justice implantés sur ce territoire. L'intérêt de ce type de partenariat repose sur le fait que les centres d'archives départementales disposent dans leurs fonds d'informations très précises sur ces établissements (notamment dans les séries U Justice et $\mathrm{Y}$ Établissements pénitentiaires). Cette dimension collaborative est essentielle à la bonne réalisation de ce projet car l'effectif du CLAMOR est insuffisant pour pouvoir en venir à bout seul. À ce propos, nous envisageons d'organiser prochainement une campagne de sensibilisation auprès des agents du ministère de la Justice afin de les inciter à participer à HUGO. L'idéal étant que ceux qui animent aujourd'hui ces lieux de justice puissent abonder eux-mêmes cette vaste opération de collecte et de sauvegarde du patrimoine judiciaire français.

\section{Conclusion}

D'après la définition arrêtée par le Conseil international des musées en 2007, un musée est : « une institution permanente sans but lucratif au service de la société et de son 
développement ouverte au public, qui acquiert, conserve, étudie, expose et transmet le patrimoine matériel et immatériel à des fins d'études, d'éducation et de délectation ${ }^{10}{ }$.

Le Musée d'histoire de la justice de Criminocorpus, bien qu'il soit virtuel, répond en tous points à cette définition. Financé par des fonds publics, il poursuit une mission de service public et tous ses contenus sont accessibles gratuitement. Sa dimension collaborative en fait un outil au service de la communauté auquel toute personne peut contribuer à différentes échelles (mise en ligne de collection, d'article scientifique, de fiche d'établissements judiciaires, d'exposition virtuelle, etc.). Il permet également de favoriser la connaissance du patrimoine judiciaire en le diffusant auprès d'un très large public et de le sensibiliser aux questions de société soulevées par la justice et son usage.

Les expériences menées sur Criminocorpus ont permis de créer un espace numérique unique dont une large part a été dédiée au recueil, à la conservation et à la valorisation publique du patrimoine pénitentiaire. Cette création peut apparaître comme une réponse limitée et insuffisante au regard de la situation d'urgence que traverse aujourd'hui le patrimoine pénitentiaire mis en péril par la fermeture de nombreux établissements. Dès lors, avec plus de dix années d'expérience, on peut considérer que l'accumulation des ressources disponibles sur Criminocorpus est un appel à la création en France d'un musée présentiel consacré à l'histoire de la justice.

\section{Bibliographie}

AGENCE POUR L'IMMOBILIER DE LA JUSTICE, s.d., Maison d'arrêt de Paris la Santé. Une prison rénovée au cœur de la cité, Paris, Ministère de la justice, URL: http://www.apij.justice.fr/ (consulté le 02.01.2018).

ARLAUD J., CARPENTIER C., LOUVEAU C., MERCIER A., RENNEVILlE M., VIMONT J.-C., 2015, Maison d'arrêt du Havre, Musée Criminocorpus, URL: https://criminocorpus.org/fr/ref/144/1/ (consulté le 02.01.2018).

BERGÉ P. \& associés, Bibliothèque Philippe Zoummeroff, crimes et châtiments, livres, manuscrits, photographies, dessins, Paris, P. Bergé \& associés, 2016.

BERGÉ P. \& associés, Bibliothèque Philippe Zoummeroff, des délits et des peines, livres, manuscrits, photographies, dessins, Paris, P. Bergé \& associés, 2014, URL : http://www.pbaauctions.com/html/index.jsp?id=18549\&lng=fr\&npp=1000 (consulté le 28/02/2018).

BERTRAND E., 2016, L'ancienne maison d'arrêt de Bourg-en-Bresse (AIN), Histoire pénitentiaire, 11, Criminocorpus. Carnet de l'histoire de la justice, des crimes et des peines, URL : http://criminocorpus.hypotheses.org/19315 (consulté le 02.01.2010).

10 http://icom.museum/la-vision/definition-du-musee/L/2/ 
BOURDEAU M., PIERRE M., 2017a, Le camp de la transportation de Saint-Laurent-duMaroni, Musée Criminocorpus, URL : https://criminocorpus.org/fr/ref/25/18653/ (consulté le 02.01.2018).

BOURDEAU M., PIERRE M., 2017b, Saint-Laurent-du-Maroni, De la préservation d'une mémoire à sa mise en valeur, Histoire pénitentiaire, 11, URL : http://criminocorpus.hypotheses.org/18879 (consulté le 28/02/2018).

BRETON J.-L., BUlME L., FARCY J.-C., PITTE D., POULAIN F., POUVREAU B., RENNEVILLE M., SANCHEZ J.-L., TRONEL J., VEYRET M., VIMONT J.-C., ZAIDMAN S., 2014, La mémoire des murs, Musée Criminocorpus, URL: https://criminocorpus.org/fr/ref/25/17629/ (consulté le 02.01.2018).

CARLIER C., RENNEVILLE M., 2007, Histoire des prisons en France - De l'Ancien Régime à la Restauration, Musée Criminocorpus, URL : https://criminocorpus.org/fr/ref/25/16933/ (consulté le 02.01.2018).

CARLIER C., RENNEVILlE M., 2007, Histoire des prisons en France - De la Monarchie de Juillet à 1914, Musée Criminocorpus, URL : https://criminocorpus.org/fr/ref/25/16934/ (consulté le 02.01.2018).

CARLIER C., RENNEVILLE M., 2007, Histoire des prisons en France - De 1914 à 2000, Musée Criminocorpus, URL: https://criminocorpus.org/fr/ref/25/16935/ (consulté le 02.01.2018).

CARLIER C., RENNEVILlE M., 2009, Histoire des prisons en France - Chronologie complémentaire (2000-2008), Musée Criminocorpus, URL: https://criminocorpus.org/fr/ref/25/16936/ (consulté le 02.01.2018).

DIRECTION DES AFFAIRES CULTURELLES D'ÎLE-DE-FRANCE, MUSÉE CARNAVALET, PARIS-MUSÉES, 2010, L'impossible photographie : prisons parisiennes, 1851-2010, Paris, Paris-Musées.

DUMAS M., 1980, L’archéologie industrielle en France, Paris, Robert Laffont.

ELKE S., 2017a, Le pénitencier d'Eastern State, Musée Criminocorpus, URL : https://criminocorpus.org/fr/ref/25/18631/ (consulté le 02.01.2018).

ELKE S., 2017b, Le site historique du pénitencier d'Eastern State, Philadelphie, Pennsylvanie, États-Unis, Histoire pénitentiaire, 11, URL: http://criminocorpus.hypotheses.org/26007 (consulté le 28/02/2018).

FAURE S., 2014, Les prisons font aussi partie de notre patrimoine, Libération, URL: http://www.liberation.fr/societe/2014/09/18/les-prisons-font-aussi-partie-de-notrepatrimoine_1103194 (consulté le 02.01.2018). 
GAUME P., SANCHEZ J.-L., VICTORIEN S., Ed., 2016, Patrimoine et architecture carcérale, Histoire pénitentiaire, 11, Criminocorpus. Carnet de l'histoire de la justice, des crimes et des peines, URL : http://criminocorpus.hypotheses.org/18809 (consulté le 14/01/2018).

GUIGNARD L., FARCY J.-C., 2015 a, Mesures policières de sûreté et populations particulièrement surveillées. Le registre des détenus administratifs de Bicêtre (1813-1851), Revue d'histoire du XIX' siècle, 50, 119-136.

GUIGNARD L., FARCY J.-C., 2015 b, La prison de Bicêtre, Musée Criminocorpus, URL : https://criminocorpus.org/fr/ref/25/17928/ (consulté le 02.01.2018).

HERBETTE L., 1891, L'œuvre pénitentiaire : études présentées à l'occasion de l'organisation du musée spécial et des expositions de l'administration française, Melun, Imprimerie administrative.

HODGKINSON S., PICHÉ J. WALBY K., WILSON J. (Eds.), 2017, The Palgrave Handbook of Prison Tourism, London, Palgrave MacMillan.

MATHURIN C., STALDER F., 2017, La maison centrale de Fontevraud, Musée Criminocorpus, URL : https://criminocorpus.org/fr/ref/25/18642/ (consulté le 02.01.2018).

MUSÉE DU CHÂTEAU DE MAYENNE, Le château de Mayenne, Musée Criminocorpus, URL : https://criminocorpus.org/fr/ref/25/18564/ (consulté le 02.01.2018).

PAPINOT E., 2018, Criminocorpus, musée d'histoire de la justice. Quels publics ? Quels usages ? Quelle ergonomie ? (Rapport de recherche), CLAMOR.

URL : https://hal.archives-ouvertes.fr/hal-01800523 (consulté le 04.08.2018).

PRADE C., 2012, L’impossible musée? 1889-2005, le musée national des Prisons, Criminocorpus. Revue Hypermédia, URL: http://journals.openedition.org/criminocorpus/1774 (consulté le 15.01.2018).

RENNEVILLE M., 2004, Pour la création d'un centre national de ressources historiques sur les crimes et les peines, Champ pénal/Penal fields, URL: http://journals.openedition.org/champpenal/document36.html (consulté le 15.01.2018).

RENNEVILLE M., 2005a, L'histoire des crimes et des peines. À propos de quelques initiatives prises par l'administration pénitentiaire (2001-2004), Revue de l'Agenais, 1, 558570.

RENNEVILLE M., 2005b, Corpus criminologique. Sciences de l'homme, traditions judiciaires et politiques pénales à la fin du XIX ${ }^{\mathrm{e}}$ siècle, Actes du colloque final Histoire des savoirs (ACI CNRS), URL : https://halshs.archives-ouvertes.fr/halshs-01390342 (consulté le 15.01.2018). 
RENNEVILLE M., 2014, SCIENCEPEINE. Sciences, savoirs et politique de l'exécution des peines en France (1911-2011). Compte-rendu final de projet. (Rapport de recherche) ANR ; Centre Alexandre Koyé-CRHST; Université de Rouen; CESDIP, URL : https://halshs.archives-ouvertes.fr/halshs-01390500/document (consulté le 15.01.2018).

RENNEVILLE M., 2014, «La « collection Zoummeroff » sur Criminocorpus », Criminocorpus (En ligne), Les sources de la recherche, Articles, mis en ligne le 16 décembre 2014, consulté le 16 avril 2018. URL : http://journals.openedition.org/criminocorpus/2913

RENNEVILLE M., 2017, Pourquoi et comment numériser des sources ?, Vingtième siècle, $133,146-150$.

RENNEVILLE M., 2018, Le renouvellement de l'histoire du droit et de la justice à travers le CLAMOR, in CLAY T., FAUVARQUE-COSSON B., RENUCCI F., ZIENTARA-LOGEAY S. (Ed.), États généraux de la recherche sur le droit et la justice, Paris, Lexis Nexis, 221-232.

RENNEVILLE M., SANCHEZ J.-L., VICTORIEN S., VIMONT J.-C., 2015, Maison d'arrêt de Paris la Santé, Musée Criminocorpus, URL : https://criminocorpus.org/fr/ref/144/2/ (consulté le 02.01.2018).

RENNEVILLE M., SANCHEZ J.-L., VICTORIEN S., 2015, Musée de l'histoire de la justice, Paris, Gobelins, l'École de l'image.

RENNEVILLE M., SANCHEZ J.-L., VICTORIEN S., 2018, Criminocorpus. Un projet numérique pour l'histoire de la justice, Digital Humanities Quaterly, 12 URL: http://digitalhumanities.org:8081/dhq/vol/12/1/000365/000365.html (consulté le 10.04.2018)

SANCHEZ J.-L., 2015, Les bagnes d'Australie, Musée Criminocorpus, URL : https://criminocorpus.org/fr/ref/25/17599/ (consulté le 02.01.2018).

SOPPELSA C., 2015, La maison d'arrêt de la Santé: une prison dans Paris, Musée Criminocorpus, URL : https://criminocorpus.org/fr/ref/25/17570/ (consulté le 02.01.2018).

VIMONT J.-C., 1993, La prison politique en France. Genèse d'un mode d'incarcération spécifique $\mathrm{XVIII}{ }^{\mathrm{e}}-\mathrm{XX}^{\mathrm{e}}$ siècles, Paris, Anthropos.

VIMONT J.-C., 2004, La prison. À l'ombre des hauts murs, Paris, Gallimard.

VIMONT J.-C., 2008, Graffiti en péril ?, Sociétés et Représentations, 25, 193-202.

VIMONT J.-C., 2012, Patrimoine carcéral des prisons françaises (sommaire), Criminocorpus. Carnet de l'histoire de la justice, des crimes et des peines, URL : https://criminocorpus.hypotheses.org/4435 (consulté le 14.01.2018). 
VIMONT J.-C., 1997, Cent mille briques. Aspects du patrimoine pénal de Haute-Normandie, Trames, 2, p. 101-112.

\section{Résumés}

Cet article présente l'action conduite par le site Criminocorpus en matière de sauvegarde du patrimoine pénitentiaire. Devenu depuis 2016 le premier musée numérique dédié à l'histoire de la justice, la question du patrimoine pénitentiaire y est bien représentée à travers différents contenus proposés dans le Musée, la revue et le carnet de recherche de Criminocorpus. Face à sa fragilité et au regard de l'urgence de la situation de ce patrimoine, Criminocorpus a œuvré pour sa préservation et sa conservation en élaborant des outils originaux (visites virtuelles d'établissements pénitentiaires, expositions virtuelles, projet collaboratif «HUGO. Patrimoine des lieux de justice », etc.).

Penitentiary Heritage in Criminocorpus’s virtual Museum (2007-2017)

This article presents the action taken by the website Criminocorpus with regard to the safeguarding of the penitentiary heritage. Since 2016, this site has become the first digital museum dedicated to the history of justice and the question of the prison patrimony is well represented through various contents declined in the Museum, the review and the research notebook of Criminocorpus. Facing its fragility and the urgency of the situation of this heritage, Criminocorpus has worked for its safeguard and preservation by developing original tools (virtual prisons visits, virtual exhibitions, collaborative project "HUGO. Heritage of places of justice", etc.). 